\title{
Contribuições da Aprendizagem Motora: a prática na intervenção em Educação Física
}

\section{Herbert UGRINOWITSCH* Rodolfo Novellino BENDA*}

*Escola de Educação Física, Fisioterapia e Terapia Ocupacional, Universidade Federal de Minas Gerais.

\begin{abstract}
Resumo
A prática é considerada o fator mais importante em aprendizagem motora e consequentemente organização da prática é o fator mais investigado na área. Existem dois diferentes tipos de prática: mental e física. Prática física é dividida em fracionamento, distribuição e variabilidade de prática. Apesar dos estudos investigarem diferentes tipos de organização da prática separadamente, em situações de ensino e treinamento todas elas interagem na organização da prática. Esta revisão também está organizada da mesma forma, mas ao final foram analisadas as possibilidades de interações.
\end{abstract}

UnITERMos: Prática mental; Organização da prática; Distribuição da prática; Fracionamento da prática.

\section{Introdução}

Atualmente, a intervenção profissional em Educação Física apresenta inúmeras possibilidades, que inclui escola, clube esportivo, academia, hospital, hotel, indústrias, ou até mesmo alunos particulares. Destas possibilidades de atuação, algumas delas têm o seu foco predominantemente em melhora de capacidades físicas (ex. "personal trainer", professores de academia); outras têm o foco de atuação na melhora de habilidades motoras (ex. técnico esportivo) e outras ainda não têm o predomínio no ensino de habilidades, mas estas fazem parte dos seus conteúdos (ex. professor de educação física escolar).

No ensino de habilidades motoras, uma área que investiga fatores que auxiliam no processo de aprendizagem destas habilidades motoras é denominada Aprendizagem Motora. Dentre os fatores investigados estão as formas de fornecer informação prévia à prática, o estabelecimento de metas a serem atingidas durante a prática, as formas de correção após a prática e, por último, a prática propriamente dita.

As formas de fornecer informação prévia à prática são a instrução verbal e a demonstração. Se por um lado a instrução verbal fornece informações sobre "o que" fazer, sendo especialmente importante para habilidades que envolvem coincidência temporal a um estímulo externo, por outro lado a demonstração fornece informaçōes sobre o "como fazer", contribuindo para a formação de um plano de ação e atuando como referência para correção durante a prática.

As metas são estabelecidas de forma genérica ou específica, podendo ainda ser de longo ou curto prazo, e todas estas podem ser de resultado, de produto ou de processo que deve ser alcançado durante a prática. Estabelecer metas parece levar o aprendiz a apresentar maior comprometimento com a tarefa, e assim executa a prática com maior dedicação e motivação.

As formas de correção após a prática estão relacionadas ao momento, quantidade e frequência de vezes que o "feedback" sobre a prática é fornecido. Parece que a participação ativa do aprendiz no processo de avaliação do ensaio realizado é importante para o processo de aprendizagem. Deste modo, o fornecimento de "feedback" pelo professor deveria complementar a avaliação do desempenho pelo aprendiz e não substituí-la.

As variáveis anteriormente citadas relacionadas à informação estão diretamente vinculadas à prática, pois informam sobre o que irá acontecer ou o que aconteceu durante a prática. Tal interpretação é reforçada na compreensão de prática como "esforço consciente de organização, execução, avaliação e modificação das açôes motoras a cada tentativa" (TANI, 1999). Assim a elaboração de um plano de ação (com base nas informaçōes prévias) como a própria avaliação por meio de 
"feedback" são consideradas inseridas na prática. Aárea da Aprendizagem Motora tem investigado diferentes formas de organizar a prática de forma a levar a uma melhor aprendizagem da habilidade praticada.

Neste artigo vamos abordar as diferentes formas de organização da prática, pois além de não serem encontrados artigos que combinem todas as suas formas de organização as demais variáveis estão diretamente relacionadas à prática. Para isso serão

\section{Prática mental}

A prática mental é entendida como a forma de imaginar a realização de uma habilidade motora na ausência de movimento (ADEGBESAN, 2009; MAGILL, 1998; RichardSON, 1967a). Neste tipo de prática acontecem ensaios internos de uma ação quando não são observados movimentos reais dos segmentos corporais envolvidos (DENIS, 1985; GENTILI, Papaxanthis \& Pozzo, 2006).

A explicação encontrada é que a prática mental auxilia na formação da imagem do movimento (Feltz \& LANDERS, 1983), a qual será utilizada para enviar os comandos para os movimentos futuros. Esta temática despertou o interesse dos pesquisadores na década de 60 (ex. Richardson, 1967a, 1967 b), pois a prática mental era vista como uma forma de ter mais eficiência no treinamento.

No geral, os resultados mostram que utilizar a prática mental quando não existe condição da prática física auxilia no processo de aprendizagem (MILLARD, Mahoney \& Wardrop, 2001; Wulf, Hosrstmann \& CHOI, 1995). Contudo, na comparação da prática mental com a prática física, a primeira não apresenta a mesma efetividade para a aprendizagem (DRISKELL, CoOper \& Moran, 1994; Feltz \& Landers, 1983).

Uma das formas como a prática mental pode contribuir no processo de aprendizagem motora é por meio de ensaios da habilidade através de imagens. A imaginação é um procedimento de prática mental no qual os indivíduos se imaginam executando uma habilidade motora (MAGILL, 1998). É usada com frequência para facilitar a aprendizagem e aperfeiçoamento de habilidades ou sequência de habilidades (OrLICK, 1986).

Pesquisadores têm trabalhado com a imaginação de duas formas: imaginar na perspectiva interna (primeira pessoa), em que o aprendiz tem a sensação de estar executando o movimento, imaginando vivenciar as sensaçôes de seu próprio corpo em uma determinada abordadas diferentes formas de prática, como a mental, pelo todo e pelas partes, a distribuição da prática e a variabilidade da prática.

A discussão sobre a prática será iniciada pela prática mental, já que ela acontece quando não há a prática física efetiva, pois quando são observados movimentos é assumido que há a prática física (MACKAY, 1981). Posteriormente serão abordadas as demais formas de organização da prática

situação; ou na perspectiva externa (terceira pessoa), em que o aprendiz vê a si mesmo ou alguém realizando o movimento, como se estivesse observando um filme, ocasião em que ele se vê na situação de um observador (DENIS, 1985; Hale, 1982). Os resultados são mais favoráveis para o uso da perspectiva interna.

Os estudos nesta temática também investigaram a posição da prática mental dentro do período total de prática, ou seja, se o efeito da prática mental seria diferente quando ela acontece antes ou após a prática física. Dos estudos realizados, foram encontrados somente dois estudos nos quais a prática mental antes da prática física levou a melhor desempenho nos testes (Etnier \& Landers, 1996; Millard, Mahoney \& Wardrop, 2001). Contudo, este efeito ainda precisa ser mais investigado, pois em todos os estudos o grupo de prática mental teve uma familiarização com a tarefa ou participou do pré-teste antes de começar o experimento (GOMES, 2009).

Outra questão que começou a ser investigada e, consequentemente, os profissionais ainda não possuem subsídios para saber dos seus efeitos na intervenção é se a prática mental auxilia na adaptação a diferentes variações, como as que acontecem em jogos esportivos coletivos e em confrontos individuais. Além disso, as variaçóes podem acontecer de forma previsível ou imprevisível (esperada ou inesperada para o executante). Esta questão começou a ser investigada por Gomes, BENDA, SCOTTI e UGRINOWITSCH (em fase final de preparação), e os resultados mostraram que a prática mental auxilia na adaptação quando as mudanças acontecem de forma previsível do que quando ela é imprevisível e já é possível planejar anteriormente da mudança apresentada.

Em resumo, os resultados dos estudos mostram que utilizar a prática mental pode ser útil quando não é possível utilizar a prática física. Neste caso, é possível utilizá-la quando um atleta está afastado 
por lesão e não pode participar dos treinamentos ou ainda no momento entre uma execução e outra em que um iniciante aguarda sua vez de praticar.
Contudo, a prática mental isolada não é tão eficiente quanto ela é quando combinada à prática física, o assunto que será abordado a seguir.

\section{Prática física}

Existem basicamente três temas investigados em relação à prática física: a fragmentação da prática, o espaçamento da prática e a variabilidade da prática. A fragmentação da prática é a divisão de uma habilidade a ser ensinada por partes (NAYLOR \& Briggs, 1963). O espaçamento da prática é o tempo de intervalo entre uma execução e outra ou ainda entre uma sessão de prática e outra (LEE \& Genovese, 1989), e por último a variabilidade da prática é a forma de variar a prática (SHEA \& MorGAN, 1979). A seguir serão discutidas cada uma destas formas de organizar a prática.

\section{Fragmentação da prática}

A aquisição de uma habilidade motora está associada à aprendizagem de cada componente dessa habilidade e da interação que existe entre esses componentes. Considerando-se que a aprendizagem da habilidade remete a um dispêndio de tempo de prática, diferentes formas de praticar podem gerar resultados distintos. Uma das formas de organizar a prática é pelo todo ou pelas partes (MAGILL, 1998), as quais também são dois extremos dentro de um "continuum" e, consequentemente, são as mais investigadas. Para isso, a análise dos conceitos de organização e complexidade (NAYLOR \& BRIGGS, 1963) da tarefa a ser aprendida seriam indicativos sobre qual seria o melhor método para a sua aprendizagem.

A complexidade refere-se ao número de partes ou de componentes de uma habilidade, bem como o processamento de informação demandado para a sua execução. A organização de uma habilidade refere-se à forma como as partes ou componentes da habilidade interagem (NAYLOR \& BRIGGS, 1963). Nesta linha de raciocínio, quanto mais componentes a habilidade tiver maior a sua complexidade e quanto maior a interação destes componentes maior a sua organização.

A adoção deste referencial indica que para habilidades de alta complexidade (número elevado de componentes) e baixa organização (pouca interação entre os componentes) o método das partes é o mais adequado. Se há alta complexidade e a interação é facilmente obtida, logo se torna importante a aprendizagem de cada uma das partes, visto que ajuntar não é o problema. No outro extremo, quando a habilidade tem de baixa complexidade e alta organização o método do todo seria o mais eficiente (NAYLOR \& BRIGGS, 1963). Com poucos componentes não é necessária a prática exclusiva para cada um deles e com alta organização, o complicador da tarefa seria encontrar o único modo de interagir os poucos componentes. Logo deve-se preocupar em promover a prática voltada para o foco principal a ser solucionado pelo aprendiz, isto é, como unir os componentes. Tradicionalmente os estudos têm investigado a questão quando as duas principais categorias de análise (complexidade e organização) estão em condições opostas. Como curiosidade, é possível ainda especular que a condição baixa complexidade e alta organização poderia ser praticada pelo todo, pois se não há tantos componentes na tarefa, ela já poderia ser praticada no seu formato final, ou seja, pelo todo. Uma condição de alta complexidade e baixa organização parece ser improvável de ser verificada em contextos reais, pois não se encontra uma tarefa com tais características.

Segundo PúBlio e TANi (1993), as características de complexidade e organização de uma habilidade podem ser entendidas como uma continuidade. Nas extremidades são colocados, respectivamente, os valores de alta e baixa, sendo que diferentes habilidades podem ser localizadas em qualquer ponto desta continuidade em função de seus atributos inerentes. Dessa forma, é possível pensar em habilidades que se encontrem em faixas intermediárias entre estes dois contínuos da complexidade e da organização.

Ainda seguindo este referencial, nas posições intermediárias utilizar métodos combinados, tais como o método por adição (FIGURA 1). Neste método, os componentes podem ser incluídos gradativamente; ou seja, após aprender o primeiro componente da habilidade o segundo componente é incluído e os dois componentes são executados. Os demais componentes a serem incluídos seguem a mesma proposta. 


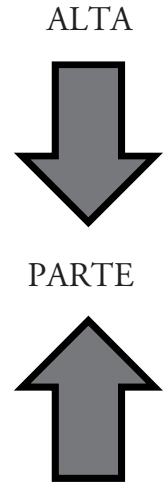

BAIXA

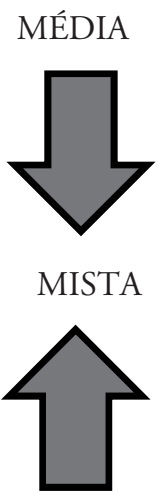

MÉDIA

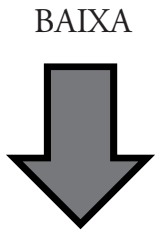

TODO

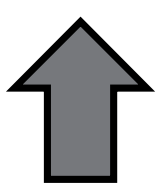

ALTA

\section{ORGANIZAÇÃO}

FIGURA 1 - Relação entre nível de complexidade e organização e tipos de prática.

No presente artigo, uma ressalva é realizada quanto ao que é proposto em livros-texto de Aprendizagem Motora (FAirbrother, 2010; MAgILl, 1998; SCHMIDT \& WrisberG, 2001). Eles contemplam distintos de prática (segmentação - partes progressivas, combinação, adição, simplificação) como fazendo parte da categoria prática por partes. Todavia, alguns destes tipos de prática se assemelham muito mais à prática do todo do que da prática por partes. Assim propōe-se uma nova interpretação desta classificação como um "continuum", já comentado anteriormente (FIGURA 2).

PARTES

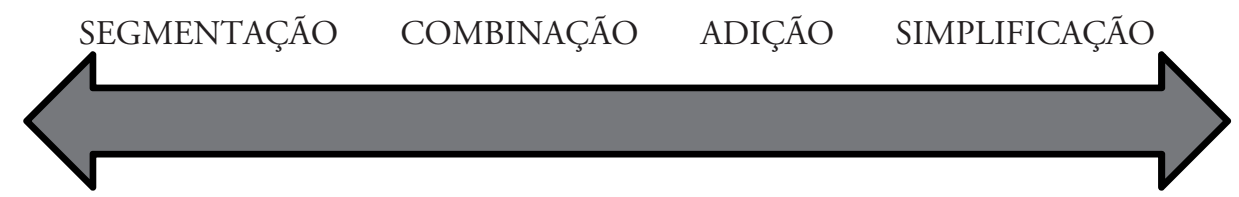

TODO

FIGURA 2 - “Continuum” entre a prática por partes, as práticas mistas e a prática pelo todo.

As pesquisas sobre esta temática são bem escassas, e a maioria delas investiga os extremos, ou seja, a prática pelo todo e pelas partes, e normalmente utilizam o mesmo referencial de NAYLOR e BRIGGS (1963) - (Hautala, 1988; Hansen, Tremblay \& Elliott, 2005; Park, Wilde \& Shea, 2004; Públio \& TANi, 1993; WatTers, 1992).

Alguns dos estudos foram realizados com tarefas de laboratório (PARK, Wilde \& SheA, 2004; Hansen, Tremblay \& Elliott, 2005) e não foi encontrada diferença entre a prática pelo todo e pelas partes. Uma possibilidade de explicação é que este referencial tem como base a diferença entre as habilidades, mas as diferenças nas tarefas de laboratório são muito pequenas para influenciar os resultados. Apesar disso, em alguns estudos o grupo que praticou por partes teve desempenho superior quando comparado ao grupo que praticou a habilidade pelo todo (NetTelbeck \& Kirby, 1976).

Outros estudos foram realizados com habilidades seriadas do dia-a-dia, e mostraram que a prática por partes levou a melhores resultados que a prática pelo todo (KNAPP \& DixON, 1952; PúBlio \& TANI, 1993). Contudo, devido ao tipo de situação experimental (os estudos foram conduzidos durante as aulas), não houve o controle da quantidade de prática de cada grupo, mas sim sobre o tempo das aulas.

Em resumo, apesar do pequeno número de estudos que investigaram esta temática, é possível identificar que com habilidades seriadas (ex. sequência de ginástica artística, coreografia de dança), o mais apropriado é utilizar o método pelas partes. Contudo, se a habilidade é discreta ou possui poucos componentes, a prática pelo todo pode ser a mais efetiva. 


\section{Espaçamento da prática}

Este tema é citado na literatura internacional como distribuição da prática (LEE \& GENOVESE, 1989), mas como se refere ao espaço de tempo entre uma execução e outra, adotaremos neste artigo o termo espaçamento. Este espaçamento da prática é conhecido como distribuído e maciço, que se refere ao espaço entre execuções de prática. O termo prática maciça já é comum na literatura brasileira, todavia há uma ressalva a ser apontada, pois o termo maciço é utilizado como tradução de "massed" que seria mais bem aplicado à presente temática se a tradução utilizada fosse prática concentrada. No presente artigo será mantido o termo tradicionalmente adotado no Brasil. Assim, a prática maciça é quando o tempo de prática é maior do que o de pausa; a prática distribuída é quando o tempo de prática é menor do que o de pausa (LeE \& GENOVESE, 1989; Newell, ANTONiou \& Carlton, 1988). Como esta relação depende da habilidade praticada, assumimos que o maciço e distribuído são dois extremos de um "continuum", que variam de acordo com a habilidade praticada. Por exemplo, a execução de um toque do voleibol demanda um tempo menor do que o de um arremesso com salto do handebol.

$\mathrm{O}$ espaçamento da prática teve bastante interesse dos pesquisadores nas décadas de 50/60, como os demais fatores que influenciam a aquisição de habilidades motoras (PEW, 1970). Estes estudos eram centrados na análise do desempenho e, então, eram inferidos os efeitos da variável na aprendizagem. Com o artigo de PEW (1970), os estudos passaram a investigar os processos de controle subjacentes ao movimento, o que diminuiu o número de artigos que investigam os fatores que influenciam a aquisição de habilidades motoras (TANI, 1992; UGRINOWITSCH \& BENDA, 2008). Logicamente, o mesmo aconteceu com os estudos que investigaram o espaçamento da prática.

Os resultados apresentam algumas tendências, mesmo ainda havendo diversas questôes que ainda não estão esclarecidas sobre a prática maciça e distribuída. Em alguns estudos a prática maciça levou a melhores resultados que a prática distribuída (GARCIA, MORENO, Reina, Menayo \& Fuentes, 2008; Lee \& Genovese; 1989), mas em outros foi a prática distribuída que mostrou melhores resultados (Bock, THOMAs \& Grigorova, 2005; LeE \& Genovese, 1989). Por último, existem ainda estudos que não encontraram diferença no efeito dos dois tipos de prática (CARRON, 1969; Stelmach, 1969; LeE \& GenOvese; 1989; Leite, Carvalho, Ugrinowitsch \& Benda, 2009). Um ponto ainda pouco investigado é a permanência do efeito do espaçamento da prática, apesar de CHASEY (1976) ter encontrado o melhor desempenho da prática distribuída no teste de retenção após a prática, mas no teste após oito semanas o efeito havia dissipado.

Além disso, outros resultados foram encontrados. A prática maciça mostrou resultados inferiores quando a habilidade era contínua; contudo, mostrou melhores resultados quando a tarefa era discreta (LeE \& GenOvese, 1989). Apesar destes resultados, ainda há discordância nos resultados, pois MACKAY, Morgan, Datta, Chang \& Darzi (2002) encontrou melhor efeito da prática distribuída.

Os melhores efeitos da prática distribuída não foram confirmados somente na aquisição de habilidades motoras. Na Educação, a redução do intervalo intertentativas levou a um efeito negativo no entendimento dos estudantes (BUDE, ImBOS, VAN DE Wiel \& Berger, 2011). Outros resultados são que a fadiga resultante do menor intervalo da prática distribuída influenciou somente o desempenho, mas não os testes de aprendizagem (MAGILL, 1998).

Existem alguns pontos nos delineamentos experimentais que dificultam a comparação dos resultados, tais como o uso de diferentes tarefas. Por exemplo, em tarefas que envolvem demanda visuomotora, a prática distribuída foi mais eficiente que a prática maciça (Studer, Koeneke, Blum \& Jäncke, 2010), mas não existe a comparação com habilidades que tenham diferentes demandas. Ainda existe um problema do uso de diferentes intervalos intertentativas. Por exemplo, enquanto Metalis (1985) utilizou um intervalo inter tentativas de 2,0 s. como prática distribuída, LeE e GenOvese (1989) utilizaram 0,5 s.

Em resumo, a maioria dos estudos mostra que a prática mais espaçada leva a aprendizagem superior, seja de habilidades motoras ou cognitivas. Uma das possíveis explicações para tal resultado é o processamento deficiente, o qual sugere que o resgate de informação aconteceria em função da quantidade e qualidade do processamento recebido por determinada informação, sendo que este processamento seria tanto melhor quanto mais perda houvesse desta informação entre uma execução e outra, obrigando o sistema a re-elaborar a resposta a cada tentativa (Jacoby, 1978). Contudo, estes estudos foram conduzidos com sujeitos iniciantes na habilidade motora praticada, mas não com sujeitos habilidosos. Consequentemente, os resultados apresentados dão subsídios para a utilização da prática distribuída no início da aprendizagem, mas no treinamento de alto rendimento ainda não existem resultados que fornecem subsídios para a intervenção do técnico esportivo. 


\section{Variabilidade da prática}

A prática pode ser organizada de forma constante ou com variaçōes. Estas variações podem ser de parâmetros físicos como força e direção de uma mesma habilidade ou ainda podem ser de habilidades distintas, e alguns autores se referem a este tema como interferência contextual ou organização da prática (Lai \& SHea, 1998). Este tema é o que mais recebeu atenção dos pesquisadores da área. $\mathrm{O}$ primeiro estudo do tema foi realizado sobre aprendizagem verbal (BATTig, 1966). Em 1979, Shea e Morgan conduziram o primeiro experimento com habilidades motoras e até hoje este tema continua sendo investigado. $\mathrm{O}$ efeito da interferência contextual é um fenômeno na aprendizagem e está relacionada com a ordem que as habilidades são praticadas (Lage, Alves, Oliveira, Palhares, Ugrinowitsch $\&$ BENDA, 2007) e parece ser um dos principais fatores que influencia a aquisição de habilidades motoras (FialHo, BENDA \& UGRINOWITSCH, 2006).

$\mathrm{Na}$ área de Aprendizagem Motora, a década de 80 ficou marcada por um grande número de estudos que investigou o efeito da interferência contextual. Nesta época o objetivo das pesquisas era comparar os efeitos da prática por blocos com os da prática aleatória. A prática por blocos é aquela em que uma situação é repetida diversas vezes e, posteriormente, outra situação é repetida diversas vezes (SHEA \& Morgan, 1979). O ponto que despertou interesse nesta temática é que a prática por blocos costuma ter melhor desempenho durante a prática, mas a prática aleatória tem melhor desempenho nos testes de aprendizagem. Ou seja, há uma inversão no desempenho observada nos testes. De forma geral, tal prática aleatória parece superior porque conduz a maior esforço cognitivo durante a prática (LEE, 2011).

Como nesta época os estudos testavam a prática em blocos e a prática aleatória, não havia a preocupação com outros fatores que serão citados adiante. Os estudos utilizavam tarefas de laboratório (ex. DeL Rey, 1982; Lee \& Magill, 1983). Os resultados nesta época estavam bastante controversos, pois enquanto alguns estudos confirmaram o melhor desempenho da prática aleatória nos testes de retenção e/ou transferência (LeE, Magill \& Weeks, 1985; WULF \& SCHMIDT, 1988) outros não encontraram diferença (Heuer \& SCHMidT, 1988).

O estudo de Wulf e SCHMIDT (1988) foi o primeiro a ter um delineamento diferente: eles testaram o efeito da interferência contextual variando parâmetros de uma mesma tarefa e também variando tarefas distintas. Os resultados foram inconclusivos quanto ao efeito da interferência contextual, mas mostraram pequeno efeito da especificidade da aprendizagem. Este estudo motivou Magill e Hall (1990) a fazerem uma revisão dos estudos sobre esta temática. Seus resultados tiveram um grande impacto na área, pois mostraram que os estudos estavam manipulando programas motores e parâmetros na prática aleatória e por blocos indistintamente. Além disso, também mostraram que a maioria dos estudos que variaram programas motores (diferentes habilidades) confirmou o efeito da interferência contextual. $\mathrm{O}$ mesmo não aconteceu com a maioria dos estudos que variavam os parâmetros de uma mesma habilidade.

Os resultados encontrados por MAGill e Hall (1990) tiveram influência direta na área. Três questóes passaram a ser o alvo principal dos pesquisadores: investigar o que deve ser manipulado durante a prática (programas ou parâmetros), investigar o que se aprende na prática variada (programas ou parâmetros de uma habilidade) e testar esta questão em situações mais próximas daquelas utilizadas no dia a dia. Todas aconteceram em paralelo, mas não com a mesma intensidade.

A primeira questão foi o que variar na prática variada. Para isto foram utilizadas diferentes tarefas, principalmente de laboratório, como o "timing" relativo (Lee, Wulf \& Schnidt, 1992; Sekiya, Magill, SidAWAY \& ANDERSON, 1994) ou no sequenciamento (Shea \& Titzer, 1993; Ugrinowitsch \& Manoel, 1996; Wood \& Ging, 1991; Wulf \& Schmidt, 1994). No geral, esses estudos confirmaram o efeito da interferência contextual, com exceção do estudo de Shea e Titzer (1993). A manipulação de parâmetros também foi testada com o tempo (SEKIYA et al., 1994; Wulf \& LEE, 1993). Não houve coerência nos resultados encontrados. WuLF (1992) manipulou referenciais de uma trajetória, UGRINOWITSCH e MANOEL (1996) a seleção de grupo muscular e WOOD e GING (1991) o tamanho de uma letra. Os resultados continuaram a ser inconclusivos.

A outra questão que foi investigada refere-se ao que é aprendido durante a prática com interferência contextual. Esta questão também teve início no estudo de Wulf e SCHMidT (1988) e ganhou corpo no estudo de SeKIYA et al. (2004). Outros estudos também investigaram esta questão (ex. LAI, SHEA, WulF $\&$ Wright, 2000; SekIYA \& Magill, 2000; SekIYA et al., 1994; SHEA, LAI, WRight, IMMINK \& BlacK, 2001; Wulf \& LeE, 1993). No geral os resultados indicam que a prática aleatória auxilia, principalmente, a aprendizagem de parâmetros (LAGE et al., 2007). 
Contudo, esta investigação do que é aprendido traz outra questão, que é a característica de uma pessoa habilidosa: ela precisa apresentar comportamento relativamente consistente, mas com capacidade de realizar ajustes de uma execução para outra. LAI e SHEA (1998) trouxeram a tona esta questão, que apesar de iniciar sem muita força atualmente é a principal questão investigada. Os autores voltaram a inserir a prática constante nos experimentos. Os seus resultados e de estudos posteriores mostraram que a prática constante seria a responsável pela formação de uma estrutura responsável pelo padrão de movimento (consistência), e a prática aleatória seria a responsável pela capacidade de modificação parametrização (Corrêa, Benda, Lustosa de Oliveira \& Tani, 2003; Corrê, Ugrinowitsch, Benda \& TANi, 2010b; LaGe et al., 2007; LAI et al., 2000; SHEA et al., 2001).

Uma particularidade dos estudos é que eles tiveram primeiro uma quantidade de prática constante para garantir a estabilidade do comportamento e, depois, iniciaram a prática variada. Quando esta condição foi comparada com grupos que iniciaram o experimento com prática aleatória ou prática por blocos, os resultados foram inferiores mesmo na presença de prática constante no final da prática (Corrêa, Massigli, Barros, Gonçalves, Tani \& Oliveira, 2010a; CorrêA et al., 2010b). Além disso, os resultados de LAGE et al. (2007) sugerem uma ordem na estruturação da prática: iniciar com a constante, depois a por blocos e por último a aleatória.

A verificação experimental deste referencial com habilidades do dia a dia foi realizada na maioria com habilidades esportivas. Foi utilizado o saque do "badminton" (GoOdE \& MAGILL, 1986; WRISBERG, 1991), a batida do golfe (BRADY, 1997; GOODWIN \&

\section{Conclusão}

Apesar de a prática mental auxiliar na aprendizagem de habilidades motoras, ela não mostra os mesmos resultados na comparação com a prática física. As três formas de estruturar a prática física foram apresentadas separadamente e ainda não há estudos que investigam a interação deles. Contudo, em situações de ensino e de treinamento de habilidades motoras elas "sempre" acontecem em conjunto. Um modelo desta situação está apresentado na FIGURA 3 e as sugestões a seguir são baseadas nos seus estudos separados, apesar de saber que da interação dos tipos de prática podem emergir resultados distintos.
MEEUWSEN, 1996); habilidades do voleibol (BORTOLI, Robazza, Durigon \& CARra, 1992; FrEnCH, RinK \& WERnER, 1990; MEIRA JUNIOR \& TANI, 2001; UGRINOWITSCH \& MANOEL, 1999), dentre outros. Apesar do elevado número de estudos, ainda não foi possível ter uma posição sobre o efeito da interferência contextual, pois os resultados não são consistentes. Um ponto em comum é que como os sujeitos eram iniciantes e as tarefas esportivas são complexas, variar a prática, por blocos ou de forma aleatória pode ser de grande dificuldade para o aprendiz, ao contrário do que é observado em tarefas mais simples de laboratório.

A interferência contextual foi testada com sujeitos habilidosos (Guadagnoli, Holcomb \& Weber, 1999; Hall, Domingues \& Cavazos, 1994; Hebert, Landin \& SOLMON, 1996) com variação de parâmetros. Os resultados foram favoráveis à prática aleatória. Ainda com habilidades esportivas, FialHo, BENDA e UGRINOWITSCH (2006) testaram a manipulação de programas com sujeitos habilidosos (jogadores de voleibol), e os resultados também fornecem subsídios à prática aleatória. Uma explicação pode ser a utilizada por LAI e SHEA (1998), de que os sujeitos habilidosos já tinham a estrutura de controle do movimento estável, e então a prática variada seria benéfica para os sujeitos com estas características.

Em resumo, a maioria dos estudos mostra a necessidade de ter prática constante no início da prática para aprender a estrutura do movimento e adquirir padronização do movimento. Posteriormente, a prática pode ser variada e seguindo uma ordem da menor (por blocos) para a maior interferência (aleatória). Além disso, a prática aleatória também apresentou melhores resultados com sujeitos habilidosos.

Podemos então iniciar dizendo que no ensino e no treinamento de habilidades motoras, o primeiro passo é identificar a habilidade a ser ensinada (sujeitos iniciantes) para decidir se ela será por partes ou pelo todo. Independente de qual seja, os resultados ainda indicam que é melhor iniciar a prática de forma distribuída. Por último, esta prática deve ser sem variaçôes, tanto de parâmetros quanto de programas. No outro extremo podemos falar dos sujeitos habilidosos - treinamento. Como estes sujeitos já têm o padrão de movimento estável e o treinamento visa pequenos ajustes dos membros ou melhorar o controle 
de parâmetros como força e direção, o mais indicado parece ser a prática pelo todo. Por último, os estudos ainda mostram que a prática aleatória foi a que levou a melhor desempenho. As demais possibilidades intermediárias do quadro deveriam ser utilizadas no processo entre o iniciante e o habilidoso, mas a sua ordem específica ainda não foi testada. Resta saber se em combinação os resultados encontrados em estudos isolados serão observados novamente, ou então novos resultados poderiam emergir a partir das suas interações.

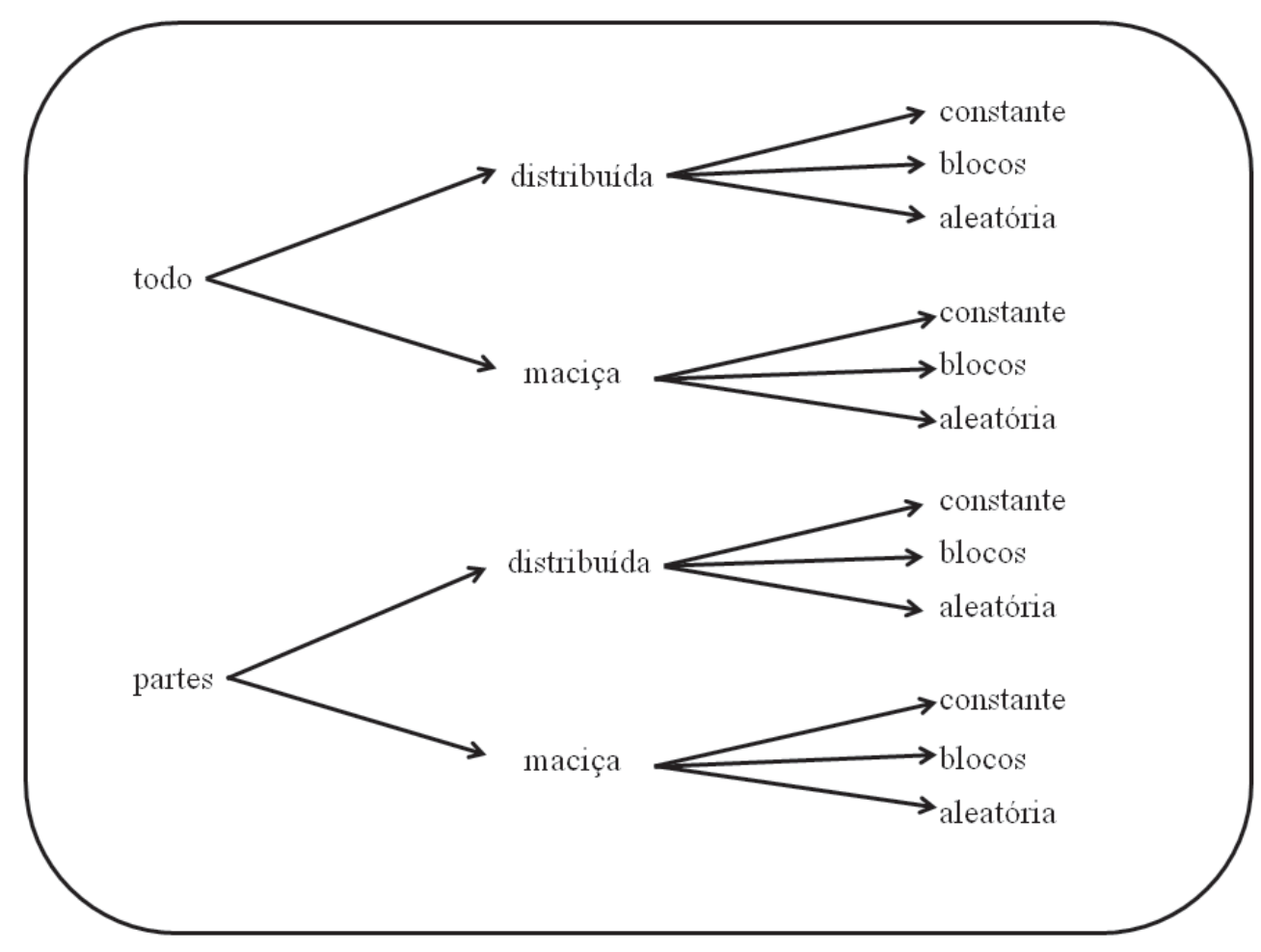

FIGURA 3 - Combinações de organização da prática no ensino de habilidades motoras.

\begin{abstract}
Contributions from Motor Learning: the practice in Physical Education intervention

Practice is considered the most important factor in motor learning and consequently practice schedule is the most investigated factor in the area. There are two different types of practice: mental and physical. Physical practice is divided in fractionated, distributed and variable. Although the studies investigated different types of practice organization separately, in teaching or training situations all of them interact in practice scheduling. This review is organized in a similar way but at the final it was analyzed the possibilities of interactions.
\end{abstract}

UnITERMS: Mental practice; Practice schedule; Practice distribution; Fractioned practice.

\title{
Referências
}

ADEGBESAN, O.A. Use of imagery by athletes in Nigeria. Perceptual and Motor Skills, Missoula, v.108, p.43-50, 2009. BATTIG, W.F. Facilitation and interference. In: BILODEUA, E.A. (Ed.) Acquisition of skill. New York: Academic Press, 1966. p.215-44.

32 • Rev. bras. Educ. Fís. Esporte, São Paulo, v.25, p.25-35, dez. 2011 N. esp. 
BOCK, O.; THOMAS, M.; GRIGOROVA, V. The effects of rest breaks on human sensorimotor adaptation. Experimental Brain Research, Berlin, v.163, p.258-60, 2005.

BORTOLI, L.; ROBAZZA, C.; DURIGON, V.; CARRA, C. Effects of contextual interference on learning technical sports skills. Perceptual and Motor Skills, Missoula, v.75, p.555-62, 1992.

BRADY, F. Contextual interference and teaching golf skills. Perceptual and Motor Skills, Missoula, v.84, p.347-50, 1997. BUDE, L.; IMBOS, T.; VAN DE WIEL, M.W.; BERGER, M.P. The effect of distributed practice on students' conceptual understanding of statistics. Higher Education, Washington, v.62, p.69-79, 2011.

CHASEY, W.C. Distribution of practice effects on learning retention and relearning by retarded boys. Perceptual and Motor Skills, Missoula, v.43, p.159-64, 1976.

CARRON, A.V. Performance and learning in a discrete motor task under massed vs. distributed practice. Research Quarterly for Exercise and Sports, Washington, v.40, p.481-9, 1969.

CORRÊA, U.C.; BENDA; R.N.; LUSTOSA de OLIVEIRA, D.; TANI, G. Practice schedule and adaptive process in the acquisition of a manual force control task. Journal of Human Movement Studies, London, v.44, p.121-8, 2003.

CORRÊA, U.C.; MASSIGLI, M.; BARROS, J.A.C.; GONÇALVES, L.A.; TANI, G.; OLIVEIRA, J.A. Constant-random practice and the adaptive process in motor learning with varying amounts of constant practice. Perceptual and Motor Skills, Missoula, v.110, p.442-52, 2010a.

CORREAA, U.C.; UGRINOWITSCH, H.; BENDA; R.N.; TANI, G. Effects of practice schedule on the adaptive process of motor learning. Revista Portuguesa de Ciências do Desporto, Porto, v.10, p.158-71, 2010b.

DEL REY, P. Effects of contextual interference on the memory of older females differing in level of physical activity. Perceptual and Motor Skills, Missoula, v.55, p.171-80, 1982.

DENIS, M. Visual imagery and the use of mental practice in the development of motor skills. Canadian Journal of Applied Sport Sciences, Champaign, v.10, p.4-6, 1985.

DRISKELL, J.E.; COPPER, C.; MORAN, A. Does mental practice enhance performance? Journal of Applied Psychology, Washington, v.79, p.481-92, 1994.

ETNIER, J.L.; LANDERS, D.M. The influence of procedural variables on the efficacy of mental practice. The Sport Psychologist, Champaign, v.10, p.48-57, 1996.

FAIRBROTHER, J.T. Fundamentals of motor behavior. Champaign: Human Kinetics, 2010.

FELTZ, D.L.; LANDERS, D.M. The effects of mental practice on motor skill learning and performance: a meta-analysis. Journal of Sport Psychology, Champaign, v.5, p.25-57, 1983.

FIALHO, J.V.A.P.; BENDA, R.N.; UGRINOWITSCH, H. The contextual interference effect in serve skill acquisition with experienced volleyball players. Journal of Human Movement Studies, London, v.50, p.65-78, 2006.

FRENCH, K.E.; RINK, J.E.; WERNER, P.H. Effects of contextual interference on retention of three volleyball skills. Perceptual and Motor Skills, Missoula, v.71, p.179-86, 1990.

GARCÍA, J.A.; MORENO, FJ.; REINA, R.; MENAYO, R.; FUENTES, J.P. Analysis of effects of distribution of practice in learning and retention of a continuous and a discrete skill presented on a computer. Perceptual and Motor Skills, Missoula, v.107, p.261-72, 2008.

GENTILI, R.; PAPAXANTHIS, C.; POZZO, T. Improvement and generalization of arm motor performance through motor imagery practice. Neuroscience, Oxford, v.137, p.761-72, 2006.

GOMES, T.V.B. Efeitos da prática mental na aquisição de habilidades motoras em sujeitos novatos: eficaz, insuficiente ou inexistente? 2009. Dissertação (Mestrado) - Escola de Educação Física, Fisioterapia e Terapia Ocupacional, Universidade Federal de Minas Gerais, Belo Horizonte, 2009.

GOMES, T.V.B.; BENDA, R.N.; SCOTTI, A.R.; UGRINOWITSCH, H. Efeitos do tipo de prática na adaptação a pertubaçôes perceptivas imprevisíveis e previsíveis. (manuscrito a ser submetido).

GOODE, S.; MAGILL, R.A. Contextual interference effects in learning three badminton serves. Research Quarterly for Exercise and Sport, Washington, v.57, p.308-14, 1986.

GOODWIN, J.E.; MEEUWSEN, H.J. Investigation of the contextual interference in the manipulation of the motor parameter of over-all force. Perceptual and Motor Skills, Missoula, v.83, p.735-43, 1996.

GUADAGNOLI, M.A.; HOLCOMB, W.R.; WEBER, T.J. The relationship between contextual interference effects and performance expertise on the learning of a putting task. Journal of Human Movement Studies, London, v.37, p.19-36, 1999. HALE, B.D. The effects of internal and external imagery on muscular and ocular concomitants. Journal of Sport Psychology, Champaign, v.4, p.379-87, 1982.

HALL, K.G.; DOMINGUES, D.A.; CAVAZOS, R. Contextual interference effects with skilled basketball players. Perceptual and Motor Skills, Missoula, v.78, p.835-41, 1994. 
HANSEN, S.; TREMBLAY, L.; ELLIOTT, D. Part and whole practice: chunking and online control in the acquisition of a serial motor task. Research Quarterly for Exercise and Sport, Washington, v.76, p.60-7, 2005.

HAUTALA, M.R. Does transfer of training help children learn juggling? Perceptual and Motor Skills, Missoula, v.67, p.563-7, 1988. HEBERT, E.P.; LANDIN, D.; SOLMON, M.A. Practice schedule effects on the performance and learning of low- and high-skilled students: an applied study. Research Quarterly for Exercise and Sport, Washington, v.67, p.52-8, 1996.

HEUER, H.; SCHMIDT, R.A. Transfer of learning among motor patterns with different relative timing. Journal of Experimental Psychology: Human Perception and Performance, Washington, v.14, p.141-52, 1988.

JACOBY, L.L. On interpreting the effects of repetition: solving a problem versus remembering a solution. Journal of Verbal Learning and Verbal Behavior, New York, v.17, p.649-67, 1978.

KNAPP, C.G.; DIXON, W.R. Learning to juggle: TI. A study ofwhole and part methods. Research Ouarterly, Washington, v.23, p.398-401, 1952.

LAGE, G.M.; ALVES, M.A.F.; OLIVEIRA, F.S.; PALHARES, L.R.; UGRINOWITSCH. H.; BENDA, R.N. The combination of practice schedules: effects on relative and absolute dimensions of the task. Journal of Human Movement Studies, London, v.52, p.21-35, 2007.

LAI, Q.; SHEA, C.H. Generalised motor program (GMP) learning: effects of reduced frequency of knowledge of results and practice variability. Journal of Motor Behavior, Washington, v.30, p.51-9, 1998.

LAI, Q.; SHEA, C.H.; WULF, G.; WRIGHT, D.L. Optimizing generalised motor program and parameter learning. Research Quarterly for Exercise and Sport, Washington, v.71, p.10-24, 2000.

LEE, T.D. Motor control in everyday actions. Champaign: Human Kinetics, 2011.

LEE, T.D.; GENOVESE, E.D. Distribution of practice in motor skill acquisition: different effects for discrete and continuous tasks. Research Quarterly for Exercise and Sport, Washington, v.59, p.277-87, 1989.

LEE, T.D.; WULF, G.; SCHMIDT, R.A. Contextual interference in motor learning: dissociated effects due to the nature of task variations. Quarterly Journal of Experimental Psychology, London, v.44A, p.627-44, 1992.

LEITE, C.M.F.; CARVALHO, M.F.S.P.; UGRINOWITSCH, H.; BENDA, R.N. Effects of distribution of practice on young adults in a complex coincident timing task. Journal of Sport \& Exercise Psychology, Champaign, v.31. p.S82-S83, 2009. MacKAY, D.G. The problem of rehearsal or mental practice. Journal of Motor Behavior, Washington, v.13, p.274-85, 1981. MacKAY, S.; MORGAN, P.; DATTA, V.; CHANG, A.; DARZI, A. Practice distribution in procedural skills training: A randomized controlled trial. Surgical Endoscopy, New York, v.16, p.957-61, 2002.

MAGILL, R.A. Motor learning: concepts and applications. Boston: WCB McGraw-Hill, 1998.

MAGILL R.A.; HALL, K.G. A review of the contextual interference effect in motor skill acquisition. Human Movement Science, Amsterdam, v.9, p.241-89, 1990.

MEIRA JUNIOR, C.M.; TANI, G. The contextual interference effect in acquisition of dart-throwing skill tested on a transfer test with extended trials. Perceptual and Motor Skills, Missoula, v.92, p.910-8, 2001.

METALIS, S.A. Effects of massed versus distributed practice on acquisition of video game skill. Perceptual and Motor Skills, Missoula, v.61, p.457-8, 1985.

MILLARD, M.; MAHONEY, C.; WARDROP, J. A preliminary study of mental and physical practice on the kayak wet exit skill. Perceptual and Motor Skills, Missoula, v.92, p.977-84, 2001.

NAILOR, J.; BRIGGS, G. Effects of task complexity and task organization on the relative efficiency of part and whole training methods. Journal of Experimental Psychology, Lancaster, v.65, p.217-44, 1963.

NETTELBECK, T.; KIRBY, N.H. A comparison of part and whole training methods with mildly mentally retarded workers. Journal of Occupational Psychology, London, v.49, n.2, p.115-20, 1976.

NEWELL, K.M.; ANTONIOU, A.; CARLTON, L.G. Massed and distributed practice effects: phenomena in search of a theory. Research Quarterly for Exercise and Sports, 59, 308-313, 1988.

ORLICK, T. Psyching for sport mental training for athletes. Champaign: Leisure Press, 1986.

PARK, J.H.; WILDE, H.; SHEA, C.H. Part-whole practice of movement sequences. Journal of Motor Behavior, Washington, v.36, p.51-61, 2004.

PEW, R.W. Toward a process-oriented theory of human skilled performance. Journal of Motor Behavior, Washington, v.2, p.8-24, 1970 .

PÚBLIO, N.S.; TANI, G. Aprendizagem de habilidades motoras seriadas da ginástica olímpica. Revista Paulista de Educação Física, São Paulo, v.7, p.58-68, 1993.

RICHARDSON, A. Mental practice: a review and discussion (Part 1). The Research Quarterly, Washington, v.38, p.95-107, 1967a.

34 • Rev. bras. Educ. Fís. Esporte, São Paulo, v.25, p.25-35, dez. 2011 N. esp. 
. Mental practice: a review and discussion (Part 2). The Research Quarterly, Washington, v.38, p.263-73, $1967 \mathrm{~b}$. SCHMIDT, R.A.; WRISBERG, C.A. Aprendizagem e performance motora: uma abordagem da aprendizagem baseada no problema. 2. ed. Porto Alegre: Artmed, 2001.

SEKYIA, H.; MAGILL, R.A. The contextual interference effect in learning force and timing parameters of the same generalized motor program. Journal of Human Movement Studies, London, v.39, p.45-71, 2000.

SEKIYA, H.; MAGILL, R.A.; SIDAWAY, B.; ANDERSON, D.I. The contextual interference effect for skill variations from the same and different generalized motor programs. Research Quarterly for Exercise and Sport, Washington, v.65, p.330-8, 1994. SHEA, C.H.; LAI, Q.; WRIGHT, D.W.; IMMINK, M.; BLACK, C. Consistent and variable conditions: effects on relative and absolute timing. Journal of Motor Behavior, Washington, v.33, p.139-52, 2001.

SHEA, J.B.; MORGAN, R.L. Contextual interference effects on the acquisition, retention and transfer of a motor skill. Journal of Experimental Psychology and Learning, Washington, v.5, p.179-87, 1979.

SHEA, J.B.; TITZER, R.C. The influence of reminder trials on contextual interference effects. Journal of Motor Behavior, Washington, v.25, p.264-74, 1993.

STELMACH, G.E. Efficiency of motor learning as a function of intertrial rest. Research Quarterly for Exercise and Sports, Washington, v.40, p.198-202, 1969.

STUDER, B.; KOENEKE, S.; BLUM, J.; JÄNCKE, L. The effects of practice distribution upon the regional oscillatory activity in visuomotor learning. Behavioral and Brain Functions, London, v.6, p.8-10, 2010.

TANI, G. Contribuições da aprendizagem motora à educação física: uma análise crítica. Revista Paulista de Educação Física, São Paulo, v.6, p.65-72, 1992.

Criança e movimento: o conceito de prática na aquisição de habilidades motoras. In: KREBS, R.J.; COPETTI, F.; BEL-

TRAME, T.S.; USTRA, M. (Eds.). Perspectivas para o desenvolvimento infantil. Santa Maria: Ediçóes SIEC, 1999. p.119-38. UGRINOWITSCH, H.; BENDA, R.N. Aprendizagem motora: produção de conhecimento e intervenção profissional. In: CORREAA, U.C. (Org.). Pesquisa em comportamento motor: a intervenção profissional em perspectiva. São Paulo: EFP/EEFEUSP, 2008. v.1, p.240-59.

UGRINOWITSCH, H.; MANOEL, E.J. Interferência contextual: manipulação de aspecto invariável e variável. Revista Paulista de Educação Física, São Paulo, v.10, p.48-58, 1996.

Interferência contextual: manipulação de programas e parâmetros na aquisição da habilidade motora saque do voleibol. Revista Paulista de Educação Física, São Paulo, v.13, p.197-216, 1999.

WATTERS, R.G. Retention of human sequence behavior following forward chaining, backward chaining, and whole task training procedures. Journal of Human Movement Studies, London, v.22, p.117-29, 1992

WOOD, C.A.; GING, C.A. The role of interference and task similarity on the acquisition, retention and transfer of simple motor skills. Research Quarterly for Exercise and Sport, Washington, v.62, p.18-26, 1991.

WRISBERG, C.A. A field test of the effect of contextual variety during skill acquisition. Journal of Teaching in Physical Education, Champaign, v.11, p.21-30, 1991.

WULF, G. Reducing knowledge of results can produce context effects in movements of the same class. Journal of Human Movement Studies, London, v.22, p.71-84, 1992.

WULF, G.; HORSTMANN, G.; CHOI, B. Does mental practice work like physical practice without information feedback? Research Quarterly for Exercise and Sport, Washington, v.66, p.262-7, 1995.

WULF, G.; LEE, T.D. Contextual interference in movements of the same class: differential effects on program and parameter learning. Journal of Motor Behavior, Washington, v.25, p.254-63, 1993.

WULF, G.; SCHMIDT, R.A. Feedback-induced variability and the learning of generalized motor program. Journal of Motor Behavior, Washington, v.26, p.348-61, 1994.

Variability in practice: facilitation in retention and transfer through schema formation or context effects? Journal of Motor Behavior, Washington, v.20, p.133-49, 1988.

ENDEREÇO

Herbert Ugrinowitsch

Escola de Educação Física, Fisioterapia e Terapia Ocupacional Universidade Federal de Minas Gerais Av. Antônio Carlos, 6627 - Pampulha 31270-901 - Belo Horizonte - MG - BRASIL e-mail: herbertu@ufmg.br 